\title{
SURGICAL MYOCARDIAL REVASCULARIZATION OUTCOMES IN KAWASAKI DISEASE: SYSTEMATIC REVIEW AND META-ANALYSIS
}

\author{
Antonio Salsano ${ }^{1}$, Jingda Liao ${ }^{2}$, Ambra Miette ${ }^{1}$, Massimo Capoccia $^{3}$, Giovanni \\ Mariscalco $^{4}$, Francesco Santini ${ }^{1}$, and Antonio Corno ${ }^{5}$ \\ ${ }^{1}$ IRCCS-San Martino IST University of Genova \\ ${ }^{2}$ Cardiovascular Research Center, University of Leicester, Leicester, U.K. \\ ${ }^{3}$ Royal Brompton and Harefield NHS Foundation Trust \\ ${ }^{4}$ Department of Surgical Sciences, Varese University Hospital, University of Insubria \\ ${ }^{5}$ Houston Children Heart Institute, Hermann Children's Hospital, University Texas Health, \\ McGovern Medical School, Houston, Texas, USA
}

September 11, 2020

\begin{abstract}
Background:Kawasaki disease (KD) is a systemic inflammatory condition occurring predominantly in children. Coronary artery bypass grafting (CABG) is performed in the presence of inflammation and aneurysms of the coronary arteries. The objectives of our study were to assess which CABG strategy provides better graft patency and long-term outcomes. Methods:A systematic review using Medline, Cochrane and Scopus databases was performed by February 2020, incorporating a network meta-analysis, performed by random-effect model within a Bayesian framework, and pooled prevalence of adverse outcomes. Hazard ratios (HR) and corresponding 95\% credible intervals (CI) were calculated by Markov chain-Monte Carlo methods. Results:Among 581 published reports, 32 studies were enrolled, including 1191 patients undergoing CABG for KD. Graft patency of internal thoracic arteries (ITA), saphenous veins (SV) and other arteries (gastroepiploic artery and radial artery) were compared. ITAs demonstrated the best patency rates at long-term follow-up (HR 0.33, 95\% CI:0.17-0.66). Pooled prevalence of early mortality after CABG was $0.28 \%$ (95\% CI:0.00-0.73\%, $\mathrm{I}^{2}=0 \%$, tau $^{2}=0$ ), with $63 / 1108$ and $56 / 1108$ patients, respectively, undergoing interventional procedures and surgical re-interventions during follow-up. Pooled prevalence was $3.97 \%$ $\left(95 \% \mathrm{CI}: 1.91-6.02 \%, \mathrm{I}^{2}=60 \%, \mathrm{tau}^{2}=0.0008\right)$ for interventional procedures and $3.47 \%$ (95\% CI:2.26-4.68\%, $\left.\mathrm{I}^{2}=5 \%, \mathrm{tau}^{2}<0.0001\right)$ for surgical re-interventions. Patients treated with arterial, venous and mixed (arterial plus second venous graft) CABG were compared to assess long-term mortality. Mixed CABG (HR 0.03,95\% CI: 0.00-0.30) and arterial CABG (HR 0.13, 95\% CI: 0.00-1.78) showed reduced long-term mortality compared with venous CABG. Conclusions:CABG in KD is a safe and effective procedure. Use of arterial conduits provides better patency rates and lower mortality at long-term follow-up.
\end{abstract}

\section{INTRODUCTION}

Kawasaki disease (KD) is a systemic inflammatory condition occurring predominantly in children ( $80 \%$ of patients are younger than 5 years of age), first reported in 1974 as an acute febrile illness with muco-cutaneous lesions and lymphadenopathy [1,2]. The incidence of KD varies across the world from 19 to 265 cases per 100,000 in children less than 5 years of age [2]. It can be complicated by a systemic vasculitis with particular involvement of the coronary arteries, if left untreated $20 \%$ of children could develop coronary artery aneurysm [2]. In the acute phase of the disease inflammatory formation of coronary aneurysms can occur, eventually associated with rupture of the latter, while thrombosis or narrowing of the affected coronary arteries can 
complicate the late phases [2-6]. In high income countries KD remains the leading cause of acquired heart disease in children, with complication such as acute myocardial infarction [2, 7-9]. Despite extraordinary results due to early recognition of the disease and treatment with intra-venous administration of immunoglobulins $[2,10]$, long-term studies showed that complications secondary to inflammation and aneurysmatic dilatation of the coronary arteries can still occur, with subsequent impairment of the left ventricular function $[2,8,9,11-14]$. Selective coronary angiography is indispensable in those situations to provide essential information for the decision making, before percutaneous or surgical myocardial revascularization [2, 14-17]. Coronary artery bypass graft (CABG) is performed in the presence of inflammation and aneurysms of the coronary arteries, using both arterial and/or venous grafts $[17,18]$. In literature only retrospective studies about CABG after KD are found and no meta-analysis has been published until now: grafts' patency and long-term outcomes such as survival rates appear uncertain. Since the risk of progression of the systemic arteritis with formation of aneurysms in other arterial districts [19], such as internal thoracic artery (ITA) [20], the choice of the best type of surgical revascularization is still a matter of discussions [18].

The objectives of our study were to assess which surgical strategy of CABG provides better graft patency and short and long-term outcomes, through a literature systematic review and a Bayesian network metaanalysis. A PICOS study design was displayed in Supplementary Table 1. Primary endpoint is graft patency for ITAs, saphenous veins (SV) and other conduits (gastroepiploic [GEA] and radial [RA] arteries). Secondary endpoints were early mortality, need for interventional procedures and surgical re-interventions, and longterm mortality for arterial, venous or mixed (arterial plus second venous graft) types of revascularization.

\section{MATERIALS AND METHODS}

No ethical approval or review protocol was applicable for this study, since it was a review of existing literature. This work was in line with PRISMA (Preferred Reporting Items for Systematic Reviews and Meta-Analyses) guidelines (Supplementary Materials).

\subsection{Search strategy}

A systematic review using Medline, Cochrane and Scopus databases have been performed by March 2020, incorporating a Bayesian network meta-analysis. A search strategy with a time interval from 1980 to 2020 was performed using the following search string for Medline (remaining strings have been reported in Supplementary Material): (((cabg[Title/Abstract]) OR bypass[Title/Abstract] OR cardiac surgery[Title/Abstract] OR revascularisation[Title/Abstract] OR revascularization[Title/Abstract])) AND kawasaki[Title/Abstract]. The articles were screened by three investigators (AS, JL, AM) initially by title and abstract, irrelevant articles were excluded, and subsequently full texts were evaluated for eligibility.

\subsection{Inclusion and exclusion criteria}

Inclusion criteria for the study were: (1) Subjects involved in the study were patients with KD who underwent surgical revascularization; (2) Comparison between different surgical strategies of myocardial revascularization; (3) At least one of the endpoints have to be included, such as graft patency, early and long-term mortality and need of interventional procedures and/or surgical re-interventions; (4) Sufficient quality data should have been provided in the original studies. Exclusion criteria for the study: (1) Case reports, conference proceedings or reviews; (2) Duplicates studies; (3) Publications with full text in languages other than English. PICOS study design was used for inclusion/exclusion criteria (Supplementary Table 1).

\subsection{Data extraction}

Three investigators (AS, JL, AM) independently identified and extracted all relevant data of eligible studies from original articles: first author's name, year of publication, numbers of patients, median age at surgery, gender, surgical strategy of myocardial revascularization, number of grafts, and endpoints such as graft patency, early and long-term mortality and need of interventional procedures and/or surgical re-interventions. Data were retrieved only from the articles, and no attempt was made to get missing data from the authors. Any disagreement was solved by consensus. 


\subsection{Quality assessment}

Study quality was assessed using the Newcastle-Ottawa Scale, a scale to evaluate the quality of nonrandomized studies [21], and the US Preventive Services Task Force [22]. The Cochrane Risk of Bias tool was also used to evaluate the methodological quality of all included studies [23]. Quality assessment of each study is presented in Supplementary Tables 2-5.

\subsection{Statistical analysis}

Baseline and early mortality data were pooled using meta-analysis of means or proportions, with individual study effect size accounted for using inverse variance methods. Results were displayed as raw values as well as percentages with $95 \%$ confidence intervals (CI) or $95 \%$ credible intervals. Graft patency and long-term mortality for arterial, venous or mixed CABG were analyzed across all arm-level studies, with direct and indirect comparisons using a mixed treatment comparison based on a Bayesian hierarchical model. HRs and corresponding 95\% credible intervals were calculated by Markov chain Monte Carlo methods using the "BUGSnet" package of R software (version 3.6.3; R Foundation, Vienna, Austria) [24]. Brooks-GelmanRubin plots method, trace plot and density plot were used to access the model convergence [25]. Besides, rank probabilities were calculated to obtain the hierarchical amount effects of multiple treatments. Given the graft type, CABG modalities and time period over which included studies were conducted, a random effects model was used. For the purposes of the mixed-treatment comparison, consistency in direct and indirect effects was assumed [26]. Heterogeneity between comparisons within the network was analyzed by examining I values for the random-effects model. Inconsistency was graphically examined using the BUGSnet nma.compare function to plot the individual data points' posterior mean deviance contributions for the consistency model versus the inconsistency model [27]. Early mortality and need of interventional procedures and surgical reinterventions were reported as pooled prevalence of adverse outcome using the package "meta" of R software [28].

\section{RESULTS}

\subsection{Search results and study characteristics}

The systematic literature review from 1981 to 2019 provided the following results: 581 reports and, after duplicates removal, 261 were screened. After screening of titles and abstract, 92 papers were excluded. The full texts of the remaining 169 articles were evaluated, and 137 studies were excluded with reasons. A total of 32 articles (observational studies and case series, no randomized controlled trial), including 1191 patients, were enrolled [29-60]. A PRISMA flow diagram of the study selection process can be found in Figure 1. The quality assessment of the included studies is reported in Supplementary Tables 2-5. Pooled patient data are presented in Table 1. All studies, patients' characteristics, main surgical strategies and early outcome results were summarized in Table 2.

\subsection{Results from network meta-analysis}

\subsubsection{Primary outcome: Graft patency}

Overall, 1191 patients were included in the present meta-analysis, with an average of 1.79 (95\% CI: 1.55-2.00) grafts per patient. ITA, RA or GEA, and SV conduits were used, however exact numbers of each graft type and graft target vessels were incompletely reported. Patency assessment at follow-up was performed using either invasive angiography or non-invasive computed tomographic angiography, and significative coronary stenosis $(>70 \%)$ or occlusions were considered non patent grafts. Of the 32 studies, 15 arm level papers were included to perform the Bayesian network meta-analysis, with a total of 1441 grafts with 324 significative stenosis/occlusions [30-33, 39, 41-44, 47, 49, 53-54, 57, 59]. Mean follow-up for coronary angiography across studies was 92.19 months (follow-up: min 3 - max 264 months). For the primary outcome we compared patency of ITAs, SV and other arteries (GEA and RA) at follow-up. Patency rates at follow-up for ITAs, SV and other arteries are $87.82 \pm 12.41 \%, 65.98 \% \pm 27.84 \%$ and $77.63 \pm 22.75 \%$, respectively. The network model, trace plot and density plot for graft patency are shown in Supplementary Figures 1 and 2. 
Summary results for all grafts patency at follow up are shown in Figure 2A, with SV used as reference. These pooled results show that ITAs and other arteries (GEA and RA) are superior to SV. Rank probability analysis for graft patency follow-up demonstrates that ITA had higher probabilities of being the first most effective treatment (Figure 2B).

The efficacy of different treatments in terms of graft patency at follow-up using HR and corresponding $95 \%$ $\mathrm{CrI}$ is displayed in Figure 3. Pairwise comparisons for graft patency at follow-up are shown in Supplementary Figures 3A, 3B and 3C. $\mathrm{I}^{2}$ demonstrated that heterogeneity was low for SV versus ITA comparison and was moderate for SV and ITA versus other arteries (GEA and RA). After comparison of consistency and inconsistency models through individual data points' posterior mean deviance contributions we conclude that there is a lack of evidence to suggest inconsistency within the network (Supplementary Figure 4).

3.2.2. Secondary outcomes: early and long-term mortality, need of interventional procedures and surgical re-interventions

In all 32 studies included in the meta-analysis only 6 early deaths were reported among 1191 patients. Pooled prevalence of early mortality after CABG was $0.28 \%$ (95\% CI: $0.00-0.73 \%, \mathrm{I}^{2}=0 \%$, tau ${ }^{2}=0$, Supplementary Figure 5).

26 studies reported interventional procedures and surgical re-interventions rates: 63/1108 and 56/1108 patients underwent interventional procedures and surgical re-interventions at follow-up (mean 110.35 months, 95\% CI: 28.50-264.00 months), respectively. Pooled prevalence of interventional procedures was 3.97\% (95\% CI: $1.91-6.02 \%, \mathrm{I}^{2}=60 \%, \mathrm{tau}^{2}=0.0008$, Supplementary Figure 6 ). Across the studies that reported interventional procedures, high heterogeneity demonstrated elevated variability across the included studies.

Pooled prevalence of surgical re-interventions was $3.47 \%$ (95\% CI: 2.26-4.68\%, $\mathrm{I}^{2}=5 \%$, $\operatorname{tau}^{2}<0.0001$, Supplementary Figure 7).

Patients treated with arterial, venous and mixed (arterial plus second venous graft) CABG were compared to assess long-term mortality. 8 arm level papers out of 32 studies were included to perform the network meta-analysis [31, 34, 39, 41-43, 49, 53]. Mean follow-up across studies for long-term mortality was 142.56 months (follow-up: min 48 - max 264 months). Survival at follow-up after arterial, venous and mixed CABG are $99.07 \pm 2.27 \%, 83.33 \% \pm 28.87 \%$ and $99.87 \pm 0.33 \%$, respectively. The network model, trace plot and density plot for long-term mortality are shown in Supplementary Figures 8 and 9. Summary results are shown in Figure 4A, while rank probability analysis are demonstrated in Figure 4B.

The efficacy of different treatments using HR and corresponding 95\% CrI is displayed in Supplementary Figure 10. Pairwise comparisons at follow-up are shown in Supplementary Figure 11 A-C. There is a lack of evidence to suggest inconsistency within the network model (Supplementary Figure 12). Briefly, mixed CABG (HR 0.03, 95\% CrI: 0.00-0.30) and arterial CABG (HR 0.13, 95\% CrI: 0.00-1.78) showed reduced long-term mortality compared with venous CABG.

More in deep, focusing the comparison between long-term mortality for Arterial CABG vs Mixed CABG we are faced with 58 single CABG out of a total of 133 cases (43.6\%) in which at least one arterial conduit has been employed(Supplementary Figure 11C) [34,39,41-42,49]. It appears that the use of arterial conduit, even better if applied in a multiple CABG setting, provides long-term mortality benefit. These findings need to be confirmed in future studies.

\section{CONCLUSIONS}

This network meta-analysis aimed to assess which CABG strategy provides better graft patency and longterm outcomes. Results from 32 studies with a total of 1191 cases demonstrated that CABG for KD is a safe procedure with satisfying long-term outcomes.

Our systematic review and meta-analysis showed a low early mortality after CABG in KD, ranging between 0 and $1 \%$. The excellent efficacy of surgical revascularization was also demonstrated by 10-year survival rates above $90 \%$, with a 3.5-4\% rate of interventional procedures or surgical re-interventions during the follow-up. 
The choice of conduits in coronary artery surgery remains a debated and controversial issue, and the following points should be considered before CABG: the growth potential of the graft relative to the somatic growth of the patient, particularly in the pediatric age; the expected long-term patency of the graft, considering that the factors that may influence long-term patency are the presence of competitive flow from native the vessel or collaterals, further development of atherosclerosis, and abnormal coronary artery structure and function at the site of the anastomosis; the potential need for future revascularization of other coronary artery branches; the choice between arterial revascularization with internal thoracic artery, free gastro-epiploic artery or free radial artery versus venous coronary artery bypass grafting with saphenous vein.

Recently published guidelines recommended a tailored approach to individual practice [61,62]. The standard surgical strategy of myocardial revascularization used in adult patients was not been adequately studied in patients with KD, giving rise to numerous speculations. The use of bilateral thoracic artery was appealing in younger patients although diabetes, obesity, chronic obstructive disease and female sex remained adverse factors and should be taken into serious consideration even in adult patients. The radial artery represented a valid alternative to the saphenous vein with encouraging medium to long-term results. The right gastroepiploic and inferior epigastric arteries remain of limited application with less supporting evidence for their usage in the adults. Allografts and artificial grafts are very rarely, if ever, used. The choice of conduit should be addressed for each patient or group of patients and balance anatomical criteria, patient background, conduit availability and surgical expertise $[18,61,62]$.

In the next future we could assist at a rising number of KD cases due to the potential association with pediatric COVID-19 [63], for which it is even more actual and important to know the better strategy of treatment for coronary complications after KD.

To assess the latter, in this study we compared graft patency of ITA, SV and other arteries (gastroepiploic artery and radial artery); and we compared patients treated with arterial, venous and mixed (arterial plus second venous graft) CABG to assess long-term mortality.

Our meta-analysis demonstrated that arterial conduits provided better patency rates at 10 years follow-up, with ITAs as the first most effective surgical options, when compared to SV. Arterial or mixed (arterial plus second venous graft) CABG, as a surrogate for the use of arterial conduits for revascularization, has been shown to be associated with higher patient's survival rates. This result complies with to the superiority of the arterial grafts when used for surgical myocardial revascularization for KD patients.

LIMITS OF THE STUDY. We have identified the following limits. Network meta-analyses for early mortality, need of interventional procedures and surgical re-interventions were not conducted. Randomized clinical trial evidences for graft patency following CABG after KD were not published. We pooled observational studies results on the topic, including not adjusted comparative studies. This is a potential source of underpowering that increases heterogeneity where there is variability between patency results. Nevertheless, this systematic review and meta-analysis represents an overview of the surgical myocardial revascularization in Kawasaki disease and may represent a starting point for further studies and refinement of the technique.

Meta-regression has not been applied. It is unlikely that this represent a source of bias since young patients with few comorbidities have been included in this review. However, it cannot certainly be excluded. Graft patency were compared regardless of the territory of revascularization. "Grey literature" was not investigated. Furthermore, as the included studies were published between 1981 and 2019, improvements in patency outcomes or long-term mortality could be expected to vary over time due to operative and therapeutic improvements.

In conclusion, our results demonstrated that CABG in KD is a safe and effective procedure, with an overall early mortality rate of $0.28 \%$, a rate of re-interventions at follow-up of $3.47 \%$ and $3.97 \%$ for interventional and surgical procedures, respectively. The use of arterial conduits was associated with better patency rates and lower mortality at follow-up.

\section{REFERENCES}


1. Kawasaki T, Kosaki F, Okawa S, Shigematsu I, Yanagawa H. A new infantile acute febrile mucocutaneous lymph node syndrome (MLNS) prevailing in Japan. Pediatrics.1974;54:271-276.

2. Newburger J W, Takahashi M, Burns J C. Kawasaki Disease. J Am Coll Cardiol.2016;67:1738-1749.

3. Fujiwara H, Hamashima Y. Pathology of the heart in Kawasaki disease. Pediatrics.1978:61;100:107.

4. Naoe, S., Shibuya, K., Takahashi, K., Wakayama, M., Masuda, H., Tanaka, M. Pathological observations concerning the cardiovascular lesions in Kawasaki disease. Cardiol Young.1991;1:212-220.

5. Kato H, Koike S, Yamamoto M, Ito Y, Yano E. Coronary aneurysms in infants and young children with acute febrile mucocutaneous lymphnode syndrome. J Pediatr.1975;86:892-898.

6. Kato H, Ichinose E, Kawasaki T. Myocardial infarction in Kawasaki disease: clinical analyses in 195 cases. J Pediatr.1986;108:923-927.

7. Hedrich CM, Schnabel A, Hospach T. Kawasaki disease. Front Pediatr.2018;6:198.

8. McCrindle BW, Rowley AH, Newburger JW, et al. Diagnosis, treatment, and long-term management of Kawasaki Disease: a scientific statement for health professionals from the American Heart Association. Circulation.2017;135:e927-e999.

9. Kato H, Sugimura T, Akagi T, et al. Long-term consequences of Kawasaki disease: a 10- to 21-year follow-up study of 594 patients. Circulation.1996;94:1379-1385.

10. De Graeff N, Groot N, Ozen S, et al. European consensus-based recommendations for the diagnosis and treatment of Kawasaki disease: the SHARE initiative. Rheumatology (Oxford).2019;58:672-682.

11. Kitamura S, Kawashima Y, Kawachi K, Fujino M, Kozuka T. Left ventricular function in patients with coronary arteritis due to acute febrile mucocutaneous lymphnode syndrome or related diseases. Am J Cardiol.1977;40:156-164.

12. Iemura M, Ishii M, Sugimura T, Akagi T, Kato H. Long term consequences of regressed coronary aneurysms after Kawasaki disease: vascular wall morphology and function. Heart.2000;83:307-311.

13. Advani N, Sastroasmoro S, Ontoseno T, Uiterwaal CS. Long-term outcome of coronary artery dilatation in Kawasaki disease. Ann Pediatr Cardiol. 2018;11:125-129.

14. Muthusami P, Luining W, McCrindle B, van der Geest R, Riesenkampff E, Yoo SJ, Seed M, Manlhiot C, Grosse-Wortmann L. Myocardial perfusion, fibrosis, and contractility in children with Kawasaki disease. JACC Cardiovasc Imaging.2018;11:1922-1924.

15. Kuno T, Shibata A, Kodaira M, Numasawa Y. Utility of coronary computed tomographt angiography in the diagnosis and management of acute-phase adult onset Kawasaki disease. Circ J.2018;82:3106-3107.

16. Dionne A, Ibrahim R, Gebhard C, et al. Difference between persistent aneurysm, regressed aneurysm, and coronary dilation in Kawasaki disease: an optical coherence tomography study. Can J Cardiol.2018;34:1120-1128.

17. Pham V, Hemptinne Q, Grinda JM, Duboc D, Varenne O, Picard F. Giant coronary aneurysms, from diagnosis to treatment: A literature review. Arch Cardiovasc Dis.2020;113:59-69.

18. Ochi M. Review: surgical treatment of giant coronary aneurysms in pediatric patients with Kawasaki disease. Gen Thorac Cardiovasc Surg.2018;66:121-129.

19. Davis FM, Eliason JL, Ganesh SK, Blatt NB, Stanley JC, Coleman DM. Pediatric non-aortic arterial aneurysms. J Vasc Surg.2016;63:466-476.

20. Hamasaki A, Uchida T, Kuroda Y, Ishizawa A, Sadahiro M. Atypical Kawasaki disease: A patient with coronary, brain, and internal mammary arteritis. J Card Surg.2019;34:359-362.

21. E Wells GA, Shea B, O'Connell D, et al. The Newcastle-Ottawa Scale (NOS) for assessing the quality of nonrandomised studies in meta-analyses. Ottawa Hospital Research Institute. http://www.ohri.ca/programs/clinical_epidemiology/oxford.asp.

22. Harris RP, Helfand M, Woolf SH, Lohr KN, Mulrow CD, Teutsch SM, Atkins D; Methods Work Group, Third US Preventive Services Task Force. Current methods of the US Preventive Services Task Force: a review of the process. Am J Prev Med.2001;20:21-35.

23. Higgins JPT, editor; Green S, editor. Cochrane Handbook for Systematic Reviews of Interventions. Chichester, UK: John Wiley and Sons.2008.

24. Béliveau A, Boyne D.J, Slater J, et al. BUGSnet: an R package to facilitate the conduct and reporting of Bayesian network Meta-analyses. BMC Med Res Methodol.2019;19:196. 
25. H.Y. Wu, J.W. Huang, H.J. Lin, et al. Comparative effectiveness of renin-angiotensin system blockers and other antihypertensive drugs in patients with diabetes: systematic review and bayesian network meta-analysis, Bmj.2013;347:f6008.

26. Van Valkenhoef G, Lu G, De Brock B, et al. Automating network meta-analysis. Res Synth Methods.2012;3:285-299.

27. Dias S, Welton NJ, Sutton AJ, Caldwell DM, Lu G, Ades A. NICE DSU technical support document 4: inconsistency in networks of evidence based on randomised controlled trials;2014.

28. Schwarzer G, Carpenter JR, Ru“cker G. Meta-analysis with R. Berlin: Springer; 2015.

29. Matsumoto Y, Fukushima S, Shimahara Y, et al. Robotic minimally invasive direct coronary artery bypass for Kawasaki disease. Gen Thorac Cardiovasc Surg.2019; Online ahead of print.

30. Tadokoro N, Fujita T, Fukushima S, et al. Multiple Coronary Artery Bypass Grafting for Kawasaki Disease-Associated Coronary Artery Disease. Ann Thorac Surg.2019;108:799-805.

31. Jeong DS, Han W, Lee YT, et al. Coronary Artery Bypass Grafting with Arterial Grafts in Patients with Kawasaki Disease Affecting the Coronary Artery: a Korean Single-Center Study. J Korean Med Sci.2018;33:e267.

32. Beckmann E, Rustum S, Marquardt S, et al. Surgical treatment of coronary artery aneurysms. J Card Surg.2017;32:674-679.

33. Ramírez-Marroquín SE, Iturriaga-Hernández A, Calderón-Colmenero J, Benita-Bordes A, CervantesSalazar JL. Coronary Revascularization in Children at a Mexican Cardiac Center: Thirteen-Year Outcomes. World J Pediatr Congenit Heart Surg.2017;8:600-604.

34. Dionne A, Bakloul M, Manlhiot C, et al. Coronary Artery Bypass Grafting and Percutaneous Coronary Intervention after Kawasaki Disease: The Pediatric Canadian Series. Pediatr Cardiol.2017;38:36-43.

35. Jang GY, Kang IS, Choi JY, et al. Nationwide survey of coronary aneurysms with diameter $>6 \mathrm{~mm}$ in Kawasaki disease in Korea. Pediatr Int.2015;57:367-372.

36. Tsuda E, Hamaoka K, Suzuki H, et al. A survey of the 3-decade outcome for patients with giant aneurysms caused by Kawasaki disease. Am Heart J.2014;167:249-258.

37. Guo HW, Chang Q, Xu JP, Song YH, Sun HS, Hu SS. Coronary artery bypass grafting for Kawasaki disease. Chin Med J (Engl).2010;123:1533-1536.

38. Muta H, Ishii M. Percutaneous coronary intervention versus coronary artery bypass grafting for stenotic lesions after Kawasaki disease. J Pediatr.2010;157:120-126.

39. Viola N, Alghamdi AA, Al-Radi OO, Coles JG, Van Arsdell GS, Caldarone CA. Midterm outcomes of myocardial revascularization in children. J Thorac Cardiovasc Surg.2010;139:333-338.

40. Legendre A, Chantepie A, Belli E et al. Outcome of coronary artery bypass grafting performed in young children. J Thorac Cardiovasc Surg.2010;139:349-353.

41. Kitamura S, Tsuda E, Kobayashi J. et al. Twenty-five-year outcome of pediatric coronary artery bypass surgery for Kawasaki disease. Circulation.2009;120:60-68.

42. Mueller F, Knirsch W, Harpes P, Prêtre R, Valsangiacomo Buechel E, Kretschmar O. Long-term follow-up of acute changes in coronary artery diameter caused by Kawasaki disease: risk factors for development of stenotic lesions. Clin Res Cardiol.2009;98:501-507.

43. Wakisaka Y, Tsuda E, Yamada O, Yagihara T, Kitamura S. Long-term results of saphenous vein graft for coronary stenosis caused by Kawasaki disease. Circ J. 2009;73:73-77.

44. Kitamura A, Mukohara N, Ozaki N, Yoshida M, Shida T. Two adult cases of coronary artery aneurysms secondary to Kawasaki disease. Thorac Cardiovasc Surg. 2008;56:57-59.

45. Tsuda E, Fujita H, Yagihara T, Yamada O, Echigo S, Kitamura S. Competition between native flow and graft flow after coronary artery bypass grafting. Impact on indications for coronary artery bypass grafting for localized stenosis with giant aneurysms due to Kawasaki disease. Pediatr Cardiol.2008;29:266270.

46. Tsuda E, Kitamura S, Kimura K et al. Long-term patency of internal thoracic artery grafts for coronary artery stenosis due to Kawasaki disease: comparison of early with recent results in small children. Am Heart J.2007;153:995-1000.

47. Tsuda E, Kitamura S. Cooperative Study Group of Japan. National survey of coronary artery bypass 
grafting for coronary stenosis caused by Kawasaki disease in Japan. Circulation.2004;110:II61-66.

48. Yamauchi H, Ochi M, Fujii M, et al. Optimal time of surgical treatment for Kawasaki coronary artery disease. J Nippon Med Sch.2004;71:279-86.

49. Inoue T, Otaki M, Oku H, Fukuda T, Shinohara T. Follow-up study of coronary artery bypass grafting in patients with Kawasaki disease. Am Heart J.2001;142:740-744.

50. Suda Y, Takeuchi Y, Ban T, Ichikawa S, Higashita R. Twenty-two-year follow-up of saphenous vein grafts in pediatric Kawasaki disease. Ann Thorac Surg. 2000;70:1706-1708.

51. Yoshikawa Y, Yagihara T, Kameda Y, et al. Result of surgical treatments in patients with coronaryarterial obstructive disease after Kawasaki disease. Eur J Cardiothorac Surg.2000;17:515-519.

52. Mavroudis C, Backer CL, Duffy CE, Pahl E, Wax DF. Pediatric coronary artery bypass for Kawasaki congenital, post arterial switch, and iatrogenic lesions. Ann Thorac Surg.1999;68:506-512.

53. Kitamura S, Kameda Y, Seki T, et al. Long-term outcome of myocardial revascularization in patients with Kawasaki coronary artery disease. A multicenter cooperative study. J Thorac Cardiovasc Surg.1994;107:663-673.

54. Suzuki A, Kamiya T, Ono Y, Okuno M, Yagihara T. Aortocoronary bypass surgery for coronary arterial lesions resulting from Kawasaki disease. J Pediatr.1990;116:567-573.

55. Kitamura S, Kawachi K, Harima R, Sakakibara T, Hirose H, Kawashima Y. Surgery for coronary heart disease due to mucocutaneous lymph node syndrome (Kawasaki disease). Report of 6 patients. Am J Cardiol.1983;51:444-448.

56. Suma K, Takeuchi Y, Shiroma K, et al. Cardiac surgery of eight children with Kawasaki disease (mucocutaneous lymph node syndrome). Jpn Heart J.1981;22:605-616.

57. Hirose H, Kawashima Y, Nakano S et al. Long-term results in surgical treatment of children 4 years old or younger with coronary involvement due to Kawasaki disease. Circulation.1986;74:I77-81.

58. Torii S, Takeuchi Y. [Early and mid-term results of right gastroepiploic artery grafting in the children with Kawasaki disease]. Nihon Kyobu Geka Gakkai Zasshi. 1996;44:945-949.

59. Ohara K, Yagihara T, Kishimoto H, et a. [Follow-up study of coronary artery bypass grafting after Kawasaki disease-early and late postoperative evaluation]. Nihon Kyobu Geka Gakkai Zasshi.1989;37:103109.

60. Takeuchi Y, Okamura Y, Torii S, Suma K. [Comparative studies of various conduits for myocardial revascularization with Kawasaki disease]. Kyobu Geka.1992;45:671-676.

61. Aldea GS, Bakaeen FG, Pal J, et al. The Society of Thoracic Surgeons Clinical Practice Guidelines on Arterial Conduits for Coronary Artery Bypass Grafting. Ann Thorac Surg.2016;101:801-809.

62. Cheng A, Slaughter MS. How I choose conduits and configure grafts for my patients - rationales and practices. Ann Cardiothorac Surg.2013;2:527-32.

63. Jones VG, Mills M, Suarez D, et al. COVID-19 and Kawasaki disease: novel virus and novel case. Hosp Pediatr.2020;10:537-540.

FIGURES TITLES AND CAPTIONSFigure 1. Flow diagram of the literature selection process Figure 2A. Forest plot for graft patency; 2B. Sucra plot for graft patency showing rank probability analysis Figure 3. League table for graft patency Figure 4A. Forest plot for long-term mortality compared to SV; 4B. Sucra plot for long-term mortality showing rank probability analysis 

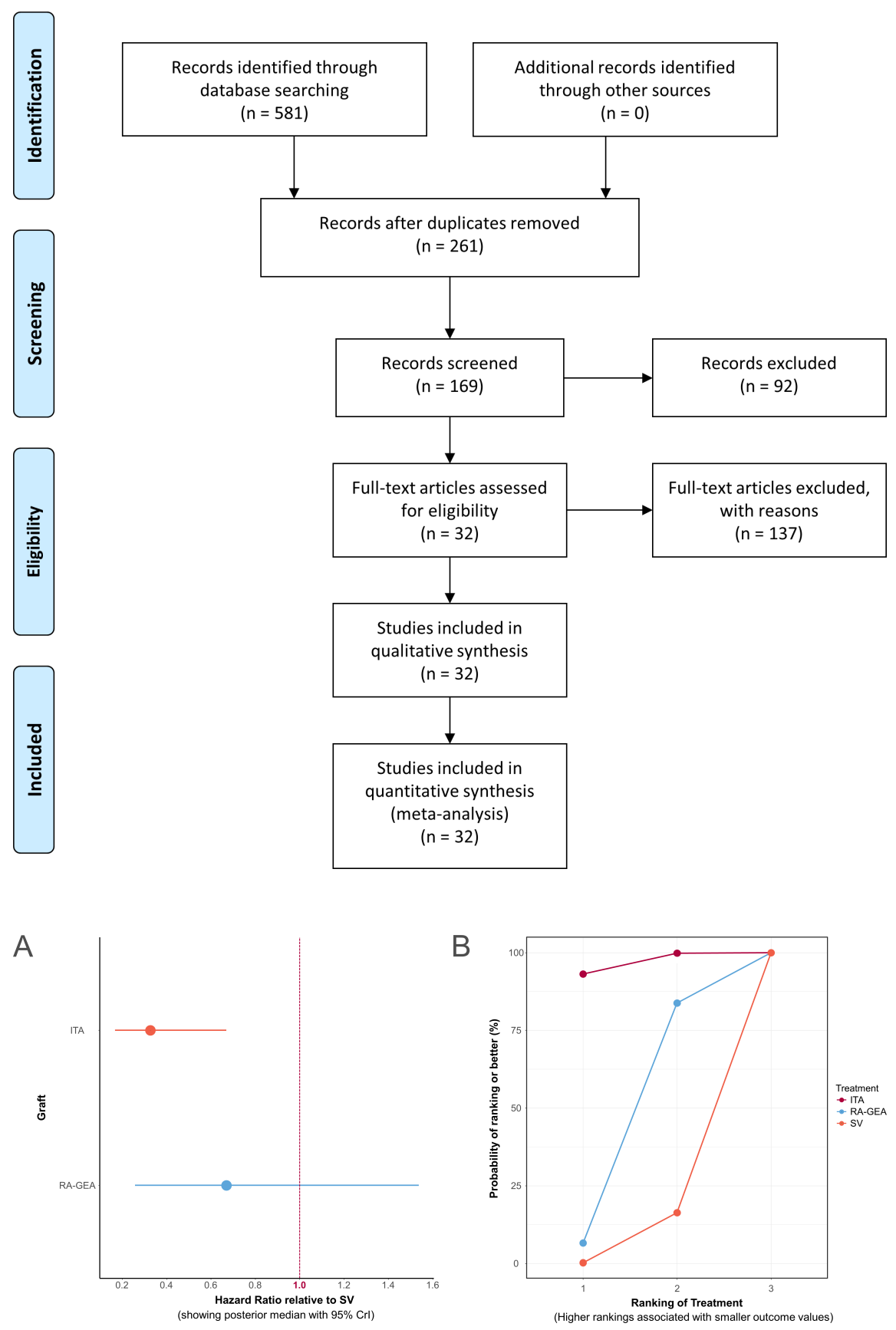

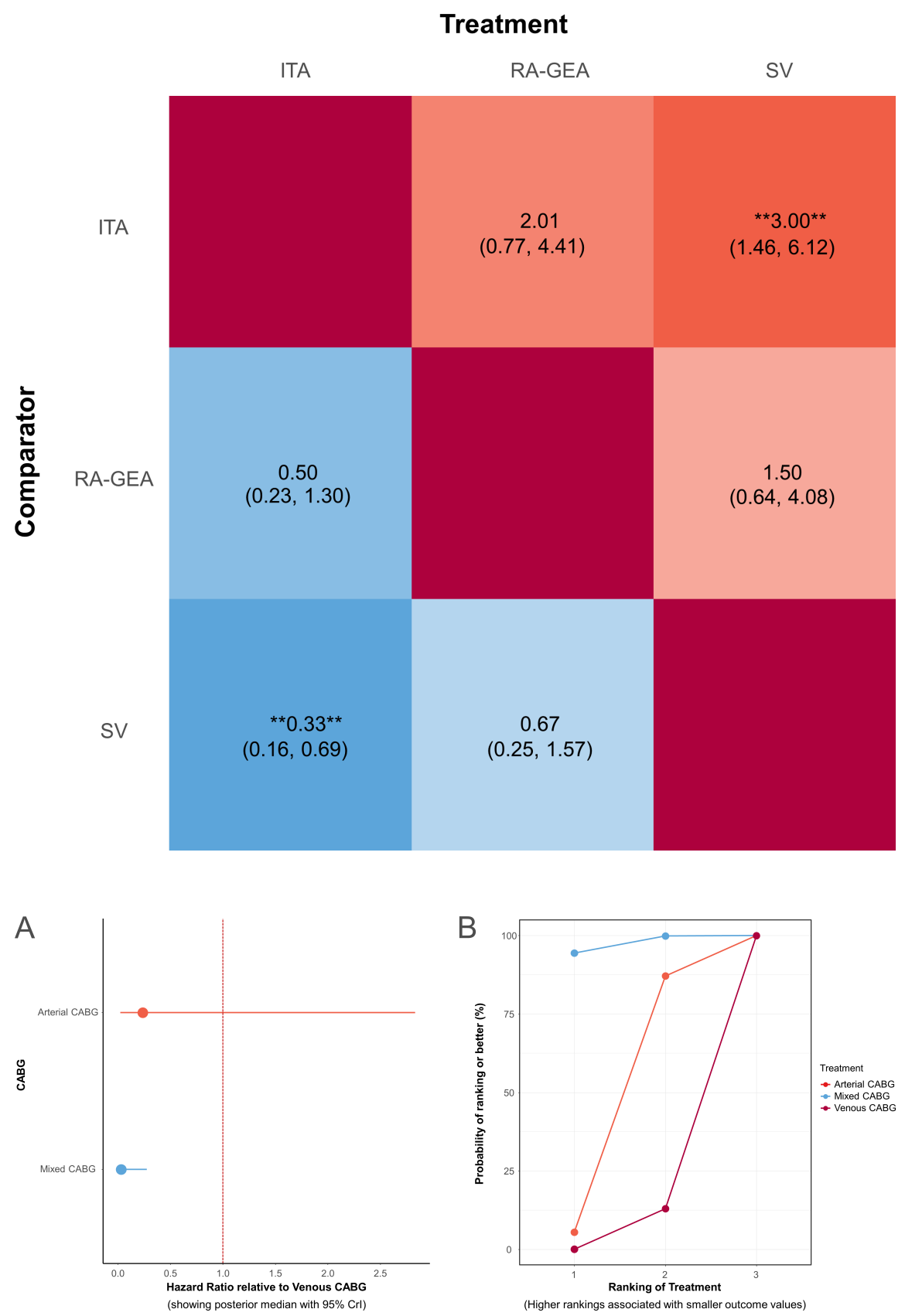

\section{Hosted file}

Table 1.docx available at https://authorea.com/users/357123/articles/479832-surgicalmyocardial-revascularization-outcomes-in-kawasaki-disease-systematic-review-and-metaanalysis

\section{Hosted file}

Table 2.docx available at https://authorea.com/users/357123/articles/479832-surgicalmyocardial-revascularization-outcomes-in-kawasaki-disease-systematic-review-and-metaanalysis 